\title{
ON INDUCTIVELY OPEN REAL FUNCTIONS
}

\author{
BIAGIO RICCERI ${ }^{1}$
}

\begin{abstract}
In this note, given a locally connected topological space $X$, we characterize those continuous and locally nonconstant real functions on $X$ which are inductively open there.
\end{abstract}

Throughout this note, $X$ denotes a locally connected topological space. Let $f$ be a real function on $X$. We recall that $f$ is said to be inductively open in $X$ (see [1]) if there exists a set $X^{*} \subseteq X$ such that $f\left(X^{*}\right)=f(X)$ and the function $f_{\mid X^{*}}: X^{*} \rightarrow f(X)$ is open.

Recently, in [2], as a consequence of a general lower semicontinuity theorem for certain multifunctions, we have

TheOREM 1 [2, ThÉorème 2.4]. Let $X$ also be connected. Then any continuous real function $f$ on $X$, such that $\operatorname{int}\left(f^{-1}(t)\right)=\varnothing$ for every $\left.t \in\right] \inf f(X)$, sup $f(X)[$, is inductively open in $X$.

It is easy to show by means of simple examples that none of the hypotheses of Theorem 1 can be dropped. In particular, this theorem is no longer true if $X$ is disconnected. Indeed, it suffices to take $X=[0,1] \cup] 2,3]$ and $f: X \rightarrow \mathbf{R}$ defined as follows:

$$
f(x)= \begin{cases}x-1 & \text { if } x \in[0,1] \\ x-2 & \text { if } x \in] 2,3]\end{cases}
$$

$f$ cannot be inductively open in $X$, since, otherwise, it would be open there, being one-to-one. But $f$ is not open in $X$ (for instance, $[0,1]$ is open in $X$ but $f([0,1])$ is not open in $f(X))$.

The aim of this note is to characterize those continuous and locally nonconstant real functions on $X$ which are inductively open there.

We first recall a lemma established in [3].

LeMma 1 [3, LemMa 3.1]. Let $S$ be a topological space, $Y$ a connected subset of $S, s_{0}, s_{1}$ two points of $Y, g$ a real function on $S$. Moreover, assume:

(1) $s_{0}$ is a local maximum (resp. minimum) point for $g$;

(2) $g\left(s_{0}\right)<g\left(s_{1}\right)\left(\right.$ resp. $\left.g\left(s_{0}\right)>g\left(s_{1}\right)\right)$;

(3) $g$ is continuous at every point of $Y$.

Then there exists $s^{*} \in Y$ with the following properties:

(i) $g\left(s^{*}\right)=g\left(s_{0}\right)$;

(ii) $s^{*}$ is not a local maximum (resp. minimum) point for $g$;

(iii) $s^{*}$ is not a local minimum (resp. maximum) point for $g$, provided that for every open set $\Omega \subseteq S$, with $\Omega \cap Y \neq \varnothing$, there exists $\bar{s} \in \Omega$ such that $g(\bar{s}) \neq g\left(s_{0}\right)$.

Received by the editors April 19, 1983.

1980 Mathematics Subject Classification. Primary 54C10, 54C30; Secondary 54D05.

${ }^{1}$ Supported by M.P.I. 
Now, we can prove

THEOREM 2. Let $f$ be a continuous real function on $X$ such that for every connected component $\Gamma$ of $f(X)$ and every $t \in \operatorname{int}(\Gamma)$ the set int $\left(f^{-1}(t)\right)$ is empty.

Then the following are equivalent:

(1) The function $f$ is inductively open in $X$.

(2) For every $t \in f(X)$ there exists a connected set $X_{t} \subseteq X$ such that $t$ belongs to the interior of $f\left(X_{t}\right)$ in $f(X)$.

Proof. Let us show that $(1) \Rightarrow(2)$. As $f$ is inductively open in $X$, there exists $X^{*} \subseteq X$ such that $f\left(X^{*}\right)=f(X)$ and $f_{\mid X^{*}}: X^{*} \rightarrow f(X)$ is open. Let $t \in f(X)$. Choose $x \in X^{*}$ such that $f(x)=t$. Since $X$ is locally connected at $x$, there is, in particular, a connected neighbourhood $X_{t}$ of $x$. Thus, with obvious meaning of the symbols, we have

$$
t \in \operatorname{int}_{f(X)}\left(f\left(X_{t} \cap X^{*}\right)\right) \subseteq \operatorname{int}_{f(X)}\left(f\left(X_{t}\right)\right)
$$

so (2) follows.

Now let us show that $(2) \Rightarrow(1)$. Put $E=\{x \in X: x$ is a local extremum point for $f\}, \tilde{X}=\{x \in X: f(x)$ is an extreme of a connected component of $f(X)$ and, for every neighbourhood $V$ of $\left.x, f(x) \in \operatorname{int}_{f(X)}(f(V))\right\}$, and $X^{*}=(X \backslash E) \cup X$. We claim $f\left(X^{*}\right)=f(X)$. Indeed, let $t \in f(X)$. By (2) there is a connected set $X_{t} \subseteq X$ such that $t \in \operatorname{int}_{f(X)}\left(f\left(X_{t}\right)\right)$. Let $x \in X_{t}$ be such that $f(x)=t$. Suppose $x \notin X^{*}$, that is, $x \in E \backslash \tilde{X}$. Let $\Gamma_{t}$ be the connected component of $f(X)$ containing $t$ and, first, assume $t$ is not an extreme of $\Gamma_{t}$. Then there exist $x_{1}, x_{2} \in X_{t}$ such that $f\left(x_{1}\right)<f(x)<f\left(x_{2}\right)$. By hypothesis the interior of $f^{-1}(t)$ is empty. Therefore, since $x$ is a local extremum point for $f$ and $f$ is continuous, by Lemma 1 , there is a point $x^{*} \in X_{t} \cap\left(f^{-1}(t) \backslash E\right)$, so $t \in f(X \backslash E) \subseteq f\left(X^{*}\right)$.

Now suppose $t$ is an extreme of $\Gamma_{t}$, for instance, the maximum of $\Gamma_{t}$. As $x \notin$ $\tilde{X}$, there exists a neighbourhood $V$ of $x$ such that $t \notin \operatorname{int}_{f(X)}(f(V))$. Hence, as $t \in \operatorname{int}_{f(X)}\left(f\left(X_{t}\right)\right)$, it follows that $f\left(X_{t}\right)$ is a nondegenerate interval contained in $\Gamma_{t}$. Applying Lemma 1 again, we then get a point $\tilde{x} \in f^{-1}(t) \cap X_{t}$ that is not a local minimum point for $f$. Thus, $\tilde{x} \in \tilde{X}$ so $t \in f(\tilde{X}) \subseteq f\left(X^{*}\right)$. A similar argument holds if $t$ is the minimum of $\Gamma_{t}$. Now we prove that the function $f_{\mid X^{*}}: X^{*} \rightarrow f(X)$ is open. Let $\Omega$ be any open subset of $X$. We must show that $f\left(\Omega \cap X^{*}\right)$ is open in $f(X)$. To this end, let $\bar{t} \in f\left(\Omega \cap X^{*}\right)$. Choose a point $\bar{x} \in \Omega \cap X^{*}$ such that $f(\bar{x})=\bar{t}$. Furthermore, let $U$ be an open connected neighbourhood of $\bar{x}$ contained in $\Omega$. Let $\Gamma_{\bar{t}}$ be the connected component of $f(X)$ containing $\bar{t}$. First suppose $\bar{t} \in \operatorname{int}\left(\Gamma_{\bar{t}}\right)$, so that, by hypothesis, we have $\operatorname{int}\left(f^{-1}(\bar{t})\right)=\varnothing$. As $\bar{x} \neq E$, the set $f(U)$ is a neighbourhood of $\bar{t}$. But, by Lemma 1 , we have $\operatorname{int}(f(U)) \subseteq f(U \backslash E) \subseteq$ $f\left(\Omega \cap X^{*}\right)$. Hence, $\bar{t}$ is an interior point of $f\left(\Omega \cap X^{*}\right)$. Now suppose $\bar{t}$ is an extreme of $\Gamma_{\bar{t}}$, for instance, the minimum of $\Gamma_{\bar{t}}$. In this case $\bar{x} \in \tilde{X}$, so $\bar{t} \in \operatorname{int}_{f(X)}(f(U))$. If $\Gamma_{\bar{t}}=\{\bar{t}\}$, then, since $f(U) \subseteq \Gamma_{\bar{t}}$, we have $U \subseteq \Omega \cap \tilde{X}$ so $\bar{t} \in \operatorname{int}_{f(X)}\left(f\left(\Omega \cap X^{*}\right)\right)$. If, on the contrary, $\Gamma_{\bar{t}} \neq\{\bar{t}\}$, then there exists $\epsilon>0$ such that $f(U) \supseteq[\bar{t}, \bar{t}+\epsilon[$. Always by Lemma 1 , we have $] \bar{t}, \bar{t}+\epsilon\left[\subseteq f(U \backslash E)\right.$ so $\left[t, \bar{t}+\epsilon\left[\subseteq f\left(\Omega \cap X^{*}\right)\right.\right.$. Hence $\bar{t} \in \operatorname{int}_{f(X)}\left(f\left(\Omega \cap X^{*}\right)\right)$. A similar argument holds if $\bar{t}$ is the maximum of $\Gamma_{\bar{t}}$.

We conclude, observing that by means of Theorem 2 it is possible to extend Theorems 2.8 and 2.10 of [4] as well as Theorem 2.2 of [5]. The details are left to the reader. 


\section{REFERENCES}

1. A. Arhangel'skii, Open and almost open mappings of topological spaces, Soviet Math. Dokl. 3 (1962), 1738-1741.

2. B. Ricceri, Sur la semi-continuité inférieure de certaines multifonctions, C. R. Acad. Sci. Paris Sér. I Math. 294 (1982), 265-267.

3. B. Ricceri and A. Villani, Openness properties of continuous real functions on connected spaces, Rend. Mat. (7) (to appear).

4. B. Ricceri, Applications de théorèmes de semi-continuité inférieure, C. R. Acad. Sci. Paris Sér. I Math. 295 (1982), 75-78.

5. _ Solutions lipschitziennes d'équations différentielles sous forme implicite, C. R. Acad. Sci. Paris Sér. I Math. 295 (1982), 245-248.

Seminario Matematico, Citta' Universitaria, Viale A. Doria 6, 95125 Catania, Italy 